\title{
Inhibición prepulso de la respuesta de sobresalto: un marcador biológico para el estudio de la salud mental en Perú
}

\author{
Prepulse inhibition of the startle response: a biological marker for the study of mental health in Peru
}

\section{Sr. Editor:}

El estrés crónico activa cascadas hormonales como la del eje hipotálamo - hipofisario - adrenal, el cual es responsable de la liberación de la hormona liberadora de corticotropina $(\mathrm{CRH})$, hormona adrenocorticotropina (ACRH) y finalmente el cortisol, además de la excesiva liberación de glutamato. Estas hormonas y neurotransmisores provocan alteraciones en el sistema nervioso central, como la excitotoxicidad, las cuales desencadenan trastornos psiquiátricos caracterizados por presentar un déficit en la inhibición prepulso (IPP) del reflejo de sobresalto (RSS) ${ }^{(1)}$.

El RSS es una respuesta incondicionada que tiene lugar inmediatamente después de la exposición a un estímulo intenso por cualquier modalidad sensorial, este reflejo consiste en la contracción de los músculos esqueléticos y faciales. Por otra parte, la IPP es un paradigma experimental que consiste en la exposición a un estímulo de baja intensidad (prepulso) y luego de $30-500 \mathrm{~ms}$ se presenta un estímulo más intenso (pulso) ${ }^{(2)}$, lo que produce, en condiciones normales, una reducción del RSS, esto permite la evaluación de funciones, estructuras y circuitos reflejos que participan en la respuesta de sobresalto ${ }^{(3)}$. La RSS es una herramienta útil por muchas razones, quizás la más importante es que proporciona una medida objetiva de la respuesta de un organismo a un contexto emocional particular, un contexto perceptual o un estímulo estresor ${ }^{(4)}$.
Los déficits del IPP se relacionan con la enfermedad de Alzheimer (EA) y con el deterioro cognitivo leve, e incluso se ha planteado la hipótesis de que puede considerarse como un marcador biológico para los cuadros mencionados ${ }^{(3)}$, el riesgo de padecer psicosis (5), el trastorno bipolar ${ }^{(2)}$, con el efecto de las drogas, como la cocaína ${ }^{(6)}$ y cannabidiol ${ }^{(7)}$ y con una variedad de trastornos neuropsiquiátricos cuyo punto de unión es la anomalía en los mecanismos de inhibición sensorial, cognitivos o motores ${ }^{(3)}$, como el trastorno de estrés postraumático ${ }^{(8)}$. Además de encontrarse relación con el estado hormonal ${ }^{(4)}$.

En Perú, la IPP tiene mayor uso en estudios realizados en el campo de la biología, por ejemplo, Tume ${ }^{(9)}$ utiliza la IPP para determinar la potencial acción antipsicótica de cuatro plantas medicinales peruanas, y Granja emplea la IPP para evaluar el efecto de diferentes antipsicóticos en modelos de híper locomoción estandarizados en ratones Swiss ${ }^{(10)}$.

La mejora en los servicios de salud mental implica el abordaje biopsicosocial del usuario, ya que tradicionalmente para el estudio de los fenómenos psicológicos se utilizan técnicas como la entrevista, la observación y la aplicación de pruebas psicológicas, ahora se propone la aplicación de nuevos paradigmas que utilicen marcadores biológicos para medir el efecto de los tratamientos. Por lo anteriormente expuesto, la IPP se presenta como un método promisorio para establecer marcadores biológicos que

\footnotetext{
Escuela Profesional de Psicología, Universidad Católica de Santa María. Arequipa, Perú.

Escuela Profesional de Psicología, Universidad Católica San Pablo. Arequipa, Perú.

Docente, Magister en Docencia Universitaria y Gestión Educativa.

Docente, Máster en Psicofarmacología y Drogas de Abuso.
} 
determinen vulnerabilidad de padecer enfermedades neuropsiquiátricas o para medir el efecto de terapias psicológicas y farmacológicas en la población peruana.

\section{Luis Fernando Ramos Vargas ${ }^{1, a}$, Guillermo Saúl Escobar Cornejo ${ }^{2, b}$}

\section{Correspondencia:}

Luis Fernando Ramos Vargas

Correo electrónico: 1ramosv@ucsm.edu.pe

\section{REFERENCIAS BIBLIOGRÁFICAS}

1. Grillon C, Davis M. Effects of stress and shock anticipation on prepulse inhibition of the startle reflex. Psychophysiology. 1997; 34:511-7.

2. Mao Z, Bo Q, Li W, Wang Z, Ma X, Wang C. Prepulse inhibition in patients with bipolar disorder: a systematic review and meta-analysis. BMC Psychiatry. 2019; 19(1):282.

3. García-Sánchez F, Martínez-Gras I, RodríguezJiménez R, Rubio G. Inhibición prepulso del reflejo de respuesta de sobresalto en los trastornos neuropsiquiátricos. Rev Neurol. 2011; 53:422-32.

4. Hantsoo L, Golden CEM, Kornfield S, Grillon C, Epperson CN. Startling Differences: Using the Acoustic Startle Response to Study Sex Differences and Neurosteroids in Affective Disorders. Curr Psychiatry Rep. 2018; 20(6):40.

5. Togay B, Cikrikcili U, Bayraktaroglu Z, Uslu A, Noyan H, Ucok A. Lower prepulse inhibition in clinical high-risk groups but not in familial risk groups for psychosis compared with healthy controls. Early Interv Psychiatry. 2019; 1: 1-7.

6. Arenas MA, Caballero-Reinaldo C, Navarro-Francés CI, Manzanedo C. Efecto de la cocaína sobre la inhibición por prepulso de la respuesta de sobresalto. Rev Neurol. 2017; 65(11):507-19.

7. Saletti PG, Tomaz C. Cannabidiol effects on prepulse inhibition in nonhuman primates. Rev Neurosci. 2018; 30(1):95-105.

8. Echiverri-Cohen A, Zoellner L, Gallop R, BedardGilligan M, Jaeger J, Feeny N. Enhanced prepulse inhibition predicts treatment response in PTSD. J Depress Anxiety. 2017; 6:287. Doi: 10.4172/21671044.1000287

9. Tume-Farfán LF. Fraccionamiento guiado por bioensayo e identificación de blancos moleculares de potencial acción antipsicótica de cuatro plantas medicinales peruanas. Tesis de maestría. Lima, Perú: Universidad Peruana Cayetano Heredia; 2017. 145 pp.

10. Granja Burbano VP. Evaluación comparativa de antipsicóticos comercializados para el tratamiento de esquizofrenia en ratones Swiss, utilizando las pruebas de campo abierto e inhibición de prepulso. Tesis de Licenciatura. Lima, Perú: Universidad Peruana Cayetano Heredia; 2015. 1 pp. (Citado el 14 de Noviembre del 2019). Disponible en: http:// repositorio.upch.edu.pe/bitstream/handle/upch/288/ Resumen.pdf? sequence $=3 \&$ isAllowed $=y$

Recibido: 30/12/2019

Aceptado: 10/12/2020 